\title{
Deshidratación de Aceituna Negra (Olea europaea. L) Variedad Sevillana - Tacna
}

RESPONSABLE: MSc Ing. Enrique De Florio Ramirez

RESUMEN Se ha realizado la optimización del proceso de deshidratación con aire caliente de la aceituna negra sevillana o criolla (Olea europaea. L) Tacna, utilizando las temperaturas de 60 y 70 grados centígrados y una velocidad del aire de secado de 0,5 $\mathrm{m} / \mathrm{s}$ a $1,5 \mathrm{~m} / \mathrm{s}$. Previamente al secado, la aceituna fue sometida a una inmersión en salmuera al $10 \%$ a una temperatura de 50 grados centigrados por espacio de 10 minutos para permeabilad la piel.

Con la ayuda del software de Stagrafics 5.1 se encontró que los parámetros óptimos de secado estudiados en este trabajo son: 68 grados centigrados y una velocidad del aire de $0,5 \mathrm{~m} / \mathrm{s}$, dándonos una velocidad de secado de 0,476 Kg de agua/kg de materia seca./hora.

La apariencia aceituna deshidratada con los diferentes fue muy similar en todos los casos.
MIEMBRO: $M S c$ c. Liliana Lanchipa Bergamini

ABSTRACT It has made the optimization of the process of dehydration with hot air from the Seville or Creole black olive (Olea europaea. L) Tacna, using temperatures of 60 and 70 degrees Celsius and a drying air velocity of $0.5 \mathrm{~m} / \mathrm{s} 1.5 \mathrm{~m} / \mathrm{s}$. Previously to drying the olive was subjected to an immersion in $10 \%$ brine at 50 degrees Celsius for 10 minutes for skin permeability.

With the help of software Stagrafics found that 5.1 is optimal drying parameters studied in this work: 68 degrees Celsius and an air velocity of $0.5 \mathrm{~m} / \mathrm{s}$, giving us a drying rate of $0.476 \mathrm{~kg}$ water/ $\mathrm{kg}$ of Curtly/hour.

The appearance with different dehydrated olives was very similar in all cases.
INTRODUCCIÓN El olivo es la especie frutícola más importante del departamento de Tacna, siendo considerado este como el primer productor de dicho cultivo (Agricultura,2008).

Se tiene también que el $15 \%$ a la producción se destina para la industria del aceite de oliva y el $85 \%$ para procesamiento en aceituna de botija (Díaz, 1988).

La alternancia en la producción primaria unida a los cambios climáticos hace una combinación tal que la aceituna producida varía en calidad y cantidad en diferentes campañas.

Este año 2008 la cosecha de aceitunas en Tacna, cuyo productor principal es $\mathrm{La}$ Yarada, sobrepasó las expectativas y algunos agricultores asumen que será de una altísima cantidad, cuyas consecuencias se presentan negativas para los precios debido a la excesiva oferta. Frente a este problema surgen varias soluciones para futuros eventos similares a este y entre las que puede ser considerada la deshidratación con aire caliente de las aceitunas producidas en Tacna; por lo tanto, el objetivo del presente trabajo es encontrar una nueva forma de industrializar la aceituna de mesa optimizando los parámetros de deshidratación por aire caliente de la aceituna negra (Olea europeae).

\section{MATERIALESYMETODOS}

La investigación se realizó en los Laboratorios de Análisis de Alimentos, Análisis Sensorial y el Laboratorio de Operaciones Unitaria ubicados en la Facultad de Ingeniería en Industrias Alimentariías de la Universidad Nacional Jorge Basadre Grohmann de Tacna.

\section{Materia Prima}

Materia prima es la aceituna negra (Olea europeae L) variedad sevillana o criolla producida en Tacna.

\section{Equipos}

Estufa, cocina. Equipo de deshidratador de bandejas con medidores de temperatura y de caudal de aire de secado. Bandejas de planchas de acero inoxidable. Manga de plástico par la recirculación de aire.Balanza analítica.Baño maría, ollas

Reactivos y material de vidrio.

Solución de Salmuera $10 \%$. Otros reactivos termómetros, salinometro, material de vidrio en general. 


\section{MÉTODOS}

\section{Diagrama experimental}

El diagrama experimental seguido en esta investigación se muestra en la Figura 3. El diagrama de flujo para la deshidratación se muestra en la Fig. 4.

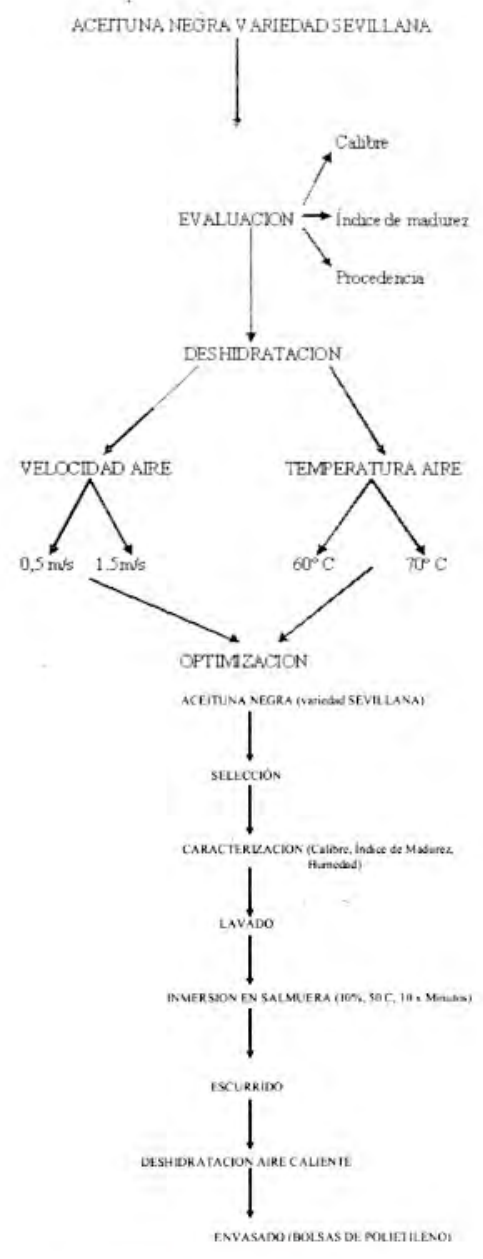

El diseño experimental para optimizar los parámetros de secado es el diseño experimental factorial de 22 , con obtención de la superficie de respuesta, obteniendo la combinación de tratamientos, teniendo en cuenta lo siguiente:

\section{Variables de entrada}

- Velocidad de aire de secado:

Valor inferior 0,5

Valor superior 1,5

- Temperatura del aire de secado:

Valor inferior $\quad 60^{\circ} \mathrm{C}$

Valor superior $70^{\circ} \mathrm{C}$

\section{Variables de Salida}

- Velocidad de Secado (gr./gr. ms. h.)

\section{RESULTADOS Y DISCUSIÓN}

Cuadros de Secado de Aceitunas.

Los resultados de la curva de secado se muestran a continuación:

Cuadro 1 Proceso de destivitratado

\begin{tabular}{|c|c|c|c|c|c|c|c|}
\hline \multicolumn{8}{|c|}{ TEMPERATURA $60^{\circ} \mathrm{C}$ Y VELOCIDAD DEL AIRE0 $5 \mathrm{M} / \mathrm{S}$} \\
\hline & & & Peso & Peso & Peso & Tiempo & Tiempe \\
\hline \multirow[t]{2}{*}{ FECHA } & HORA & PESO $|q|$ & Perdido & Perdido & Perdido & secado & secado. \\
\hline & & & $\mathrm{Gr}$ & Acum & $\%$ & Hrs & Acumul \\
\hline \multirow[t]{3}{*}{$04 / 08 / 2008$} & $10: 10$ & 476 & 0 & 0 & 0.00 & 0) & \\
\hline & $11: 58$ & 463 & 13 & 13 & 97.27 & 1.48 & 1.48 \\
\hline & $15: 00$ & 440 & 23 & 36 & 92.44 & 3.02 & 4.5 \\
\hline \multirow[t]{3}{*}{$05 / 08 / 2008$} & $11: 00$ & 420 & 6 & 56 & 88.24 & 0.47 & 4.97 \\
\hline & $12: 00$ & 418 & 2 & 58 & 87.82 & 1 & 5.97 \\
\hline & $15: 34$ & 394 & 24 & 82 & 8277 & 3.34 & 9.31 \\
\hline \multirow[t]{4}{*}{$06 / 08 / 2008$} & 11:08 & 371 & 8 & 105 & 77.94 & 2.38 & 11.69 \\
\hline & $12: 00$ & 368 & 3 & 108 & 77.31 & 0.52 & 12.21 \\
\hline & $12: 55$ & 364 & 4 & 112 & 76.47 & 0.55 & 12.76 \\
\hline & $15: 12$ & 352 & 12 & 124 & 73.95 & 2.17 & 14.93 \\
\hline \multirow[t]{2}{*}{$07 / 08 / 2008$} & $12: 18$ & 321 & 11 & 155 & 67.44 & 2.18 & 17.11 \\
\hline & $16: 00$ & 305 & 16 & 171 & 64.08 & 3.42 & 20.53 \\
\hline \multirow[t]{2}{*}{$08 / 08 / 2008$} & $11: 00$ & 281 & 15 & 195 & 59.03 & 2 & 22.53 \\
\hline & $15: 00$ & 268 & 13 & 208 & 56.30 & 4 & 26.53 \\
\hline \multirow[t]{2}{*}{$13 / 08 / 2008$} & $10: 05$ & 253 & 15 & 223 & 53.15 & 0.6 & 27.13 \\
\hline & $15: 00$ & 253 & 0 & 243 & 53.51 & 2.55 & 29.68 \\
\hline \multirow[t]{2}{*}{$14 / 08 / 2008$} & $10: 49$ & 233 & 1. & 243 & 48.95 & 1.19 & 30.87 \\
\hline & $15: 00$ & 229 & 4 & 247 & 48.11 & 4.02 & 34.89 \\
\hline \multicolumn{3}{|c|}{$\begin{array}{l}\text { Calibre }=94 \\
\text { Humedad }=50.1\end{array}$} & & & 4 & & \\
\hline
\end{tabular}

Cuadro 2..- Resultados del proceso de deshidratado temperatura $60^{\circ} \mathrm{c}$ y velocidad del aire $1.5 \mathrm{~m} / \mathrm{s}$

\begin{tabular}{|c|r|r|r|r|r|r|r|}
\hline & & & Peso & Peso & Peso & Tiempo & Tiempo \\
\hline FECHA & HORA & PESO & perdid. & perdid. & perdid. & & Acum \\
\hline & & \multicolumn{1}{|c|}{ gr } & gr & Acum. & \% & Hrs & Hrs \\
\hline $23 / 06 / 2008$ & $12: 20: 00$ & 508 & 0 & 0 & 0 & 0.00 & 0 \\
\hline & $13: 25: 00$ & 507 & 1 & 1 & 99.8 & 1.55 & 1.55 \\
\hline & $14: 10: 00$ & 499 & 8 & 9 & 98.23 & 0.50 & 2.05 \\
\hline & $15: 47: 00$ & 473 & 26 & 35 & 93.11 & 1.37 & 3.42 \\
\hline $24 / 09 / 2008$ & $9: 32: 00$ & 472 & 1 & 36 & 92.91 & 1.27 & 4.69 \\
\hline & $10: 00: 00$ & 471 & 1 & 37 & 92.72 & 3.00 & 7.69 \\
\hline & $12: 00: 00$ & 462 & 9 & 46 & 90.94 & 1.00 & 8.69 \\
\hline $24 / 09 / 2008$ & $14: 00: 00$ & 453 & 5 & 55 & 89.17 & 2.40 & 11.09 \\
\hline & $16: 20: 00$ & 420 & 33 & 88 & 82.68 & 2.00 & 13.09 \\
\hline $25 / 09 / 2008$ & $10: 00: 00$ & 409 & 9 & 99 & 80.51 & 1.25 & 14.34 \\
\hline & $11: 30: 00$ & 395 & 14 & 113 & 77.76 & 1.35 & 15.69 \\
\hline $26 / 09 / 2008$ & $10: 00: 00$ & 390 & 5 & 118 & 76.77 & 2.00 & 17.69 \\
\hline & $11: 00: 00$ & 387 & 3 & 121 & 76.18 & 1.30 & 18.99 \\
\hline & $13: 00: 00$ & 378 & 7 & 130 & 74.41 & 2.00 & 20.99 \\
\hline & $14: 40: 00$ & 368 & 10 & 140 & 72.44 & 1.40 & 22.39 \\
\hline & $16: 09: 00$ & 359 & 9 & 149 & 70.67 & 1.29 & 23.68 \\
\hline & $10: 20: 00$ & 346 & 2 & 162 & 68.11 & 2.00 & 25.68 \\
\hline $27 / 09 / 2008$ & $12: 30: 00$ & 344 & 2 & 164 & 67.72 & 2.10 & 27.78 \\
\hline & $15: 00: 00$ & 344 & 0 & 164 & 67.72 & 2.30 & 30.08 \\
\hline & & & & & & & \\
\hline Indice de Madurez $=4,72$ & & & & & & \\
\hline Humedad 64.8\% & & & & & & & \\
\hline
\end{tabular}

Cuadro 3-- Proceso de Secado A una temperatura $65^{\circ} \mathrm{c}$ y velocidad del aire $1.0 \mathrm{~m} / \mathrm{s}$

\begin{tabular}{|c|r|r|r|r|r|r|r|r|}
\hline & & & Peso & Peso & Pese & Pese & Tiemp & Tiempo \\
\hline FECHA & HORA & PESO & Perd. & Perd. & MS & Perd. & & Acum. \\
\hline dd/mm/aa & & gr & gr. & gr. Ac. & gr. & $\%$ & Hrs & Hrs \\
\hline & $11: 00$ & 489 & 0 & 0 & 0 & & 0 & 0 \\
\hline $23 / 10 / 2008$ & $11: 20$ & 488 & 1 & 1 & 489 & 99.80 & 1.55 & 1.55 \\
\hline & $13: 30$ & 469 & 19 & 20 & 489 & 95.91 & 0 & 1.55 \\
\hline & $15: 08$ & 455 & 14 & 34 & 489 & 93.05 & 1.3 & 2.85 \\
\hline $24 / 10 / 2008$ & $09: 00$ & 409 & 46 & 80 & 489 & 83.64 & 3 & 5.85 \\
\hline & $15: 20$ & 364 & 45 & 125 & 489 & 74.44 & 1 & 6.85 \\
\hline $27 / 10 / 2008$ & $08: 00$ & 358 & 6 & 131 & 489 & 73.21 & 0 & 6.85 \\
\hline & $13: 00$ & 345 & 13 & 144 & 489 & 70.55 & 2.4 & 9.25 \\
\hline & $15: 40$ & 292 & 53 & 197 & 489 & 59.71 & 2 & 11.25 \\
\hline $28 / 10 / 2008$ & $08: 30$ & 280 & 12 & 209 & 489 & 57.26 & 0 & 11.25 \\
\hline & $11: 40$ & 265 & 15 & 224 & 489 & 54.19 & 1.25 & 12.5 \\
\hline & $15: 35$ & 252 & 13 & 237 & 489 & 51.53 & 1.35 & 13.85 \\
\hline
\end{tabular}


Cuadro 4.- Resultados del proceso de deshidratado temperatura $70^{\circ}$ c y velocidad del aire $0.5 \mathrm{~m} / \mathrm{s}$

\begin{tabular}{|c|c|c|c|c|c|c|c|}
\hline & & & Peso & Peso & Peso & Tiems & Tiemp \\
\hline \multirow[t]{2}{*}{ FECHA } & HORA & PESO & Perd. & Perd. & & & Acum. \\
\hline & & ar & ar & ar. A. & $\%$ & Hirs & His \\
\hline \multirow[t]{10}{*}{$30 / 06 / 2008$} & $09: 20$ & 460 & 요 & 0 & 0.00 & 0 & 0 \\
\hline & 09:51 & 458 & 2 & 2 & 99.57 & 0.31 & 0.31 \\
\hline & $10: 20$ & 458 & 0 & 2 & 99.57 & 0.25 & 0.6 \\
\hline & $10: 50$ & 458 & 0 & 2 & 99.57 & 0.3 & 0.9 \\
\hline & $11: 20$ & 456 & 2 & 4 & 99.13 & 0.3 & 1.2 \\
\hline & $12: 30$ & 450 & 6 & 10 & 97.83 & 1.1 & 2.3 \\
\hline & $13: 00$ & 445 & 5 & 15 & 96.74 & 0.3 & 2.6 \\
\hline & $13: 30$ & 440 & 5 & 20 & 95.65 & 0.3 & 2.9 \\
\hline & $14: 00$ & 437 & 3 & 23 & 95.00 & 0.3 & 3.2 \\
\hline & $15: 10$ & 430 & 7 & 30 & 93.48 & 1.1 & 4.3 \\
\hline \multirow{7}{*}{$01 / 07 / 2008$} & $09: 30$ & 409 & 3 & 51 & 88.91 & 1.3 & 5.6 \\
\hline & $10: 00$ & 416 & -7 & 44 & 90.43 & 0.3 & 5.9 \\
\hline & $11: 00$ & 414 & 2 & 46 & 90.00 & 1 & 6.9 \\
\hline & $11: 25$ & 414 & 0 & 46 & 90.00 & 0.25 & 7.15 \\
\hline & $12: 00$ & 408 & 6 & 52 & 88.70 & 0.35 & 7.5 \\
\hline & $12: 30$ & 408 & 0 & 52 & 88.70 & 0.3 & 7.8 \\
\hline & $13: 00$ & 407 & 1 & 53 & 88.48 & 0.3 & 8.1 \\
\hline \multirow[t]{8}{*}{$02 / 07 / 2008$} & $09: 20$ & 358 & 12 & 102 & 77.83 & 1.2 & 9.3 \\
\hline & $10: 50$ & 358 & 0 & 102 & 77.83 & 1.3 & 10.6 \\
\hline & 11:30 & 356 & 2 & 104 & 77.39 & 0.4 & 11 \\
\hline & $12: 00$ & 353 & 3 & 107 & 76.74 & 0.3 & 11.3 \\
\hline & $12: 30$ & 350 & 3 & 110 & 76.09 & 0.3 & 11.6 \\
\hline & $13: 30$ & 346 & 4 & 114 & 75.22 & 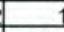 & 12.6 \\
\hline & $15: 37$ & 333 & 13 & 127 & 72.39 & 2.07 & 14.67 \\
\hline & $16: 50$ & 314 & 19 & 146 & 68.26 & 1.13 & 15.8 \\
\hline \multirow[t]{6}{*}{$03 / 07 / 2008$} & $09: 00$ & 303 & 11 & 157 & 65.87 & 0 & 15.8 \\
\hline & $10: 50$ & 296 & 7 & 164 & 64.35 & 1.5 & 17.3 \\
\hline & 11:00 & 296 & 0 & 164 & 64.35 & 0.1 & 17.4 \\
\hline & $11: 30$ & 295 & 1 & 165 & 64.13 & 0.3 & 17.7 \\
\hline & 12:00 & 294 & 1 & 166 & 63.91 & 0.3 & 18 \\
\hline & 13:00 & 286 & 8 & 174 & 62.17 & 1 & 19 \\
\hline \multicolumn{3}{|c|}{$\begin{array}{l}\text { Indice de Madurez= 4,66 } \\
\text { calibre }=108 / \mathrm{Kg}\end{array}$} & & & $31,74 \%$ & & \\
\hline
\end{tabular}

Cuadro 5.- Resultados del proceso de deshidratado

\begin{tabular}{|c|c|c|c|c|c|c|c|c|}
\hline & & & Peso & Peso & Peso & Peso & Tiempd & Tiempo \\
\hline \multirow[t]{2}{*}{ FECHA } & HORA & \begin{tabular}{|l|} 
PESO \\
\end{tabular} & Perd. & Perd. & inicial & Perd. & Secado & Acumul. \\
\hline & & & ar & or. A & & $\%$ & Hrs & Hrs \\
\hline \multirow[t]{6}{*}{$14 / 07 / 2008$} & $11: 00$ & 493 & 0 & 0 & 493 & 0.00 & 0 & 0 \\
\hline & $12: 00$ & 460 & 33 & 33 & 493 & 93.31 & 1 & 1 \\
\hline & $13: 00$ & 453 & 7 & 40 & 493 & 91.89 & 1 & 2 \\
\hline & $13: 50$ & 449 & 4 & 44 & 493 & 91.08 & 0.5 & 2.5 \\
\hline & $15: 04$ & 427 & 22 & 66 & 493 & 86.61 & 0.4 & 2.9 \\
\hline & $15: 17$ & 420 & 7 & 73 & 493 & 85.19 & 1.14 & 4.04 \\
\hline \multirow[t]{5}{*}{$15 / 07 / 2008$} & $10: 00$ & 407 & 8 & 86 & 493 & 82.56 & 2 & 6.04 \\
\hline & $10: 50$ & 389 & 18 & 104 & 493 & 78.90 & 0.5 & 6.54 \\
\hline & $13: 30$ & 376 & 13 & 117 & 493 & 76.27 & 1.6 & 8.14 \\
\hline & $15: 00$ & 340 & 36 & 153 & 493 & 68.97 & 1.3 & 9.44 \\
\hline & $09: 00$ & 330 & 5 & 163 & 493 & 66.94 & 1 & 10.44 \\
\hline \multirow[t]{3}{*}{$16 / 07 / 2008$} & $12: 00$ & 308 & 22 & 185 & 493 & 62.47 & 3 & 13,44 \\
\hline & $03: 30$ & 283 & 25 & 210 & 493 & 57.40 & 3.3 & 16.74 \\
\hline & $09: 30$ & 268 & 14 & 225 & 493 & 54.36 & 1.3 & 18.04 \\
\hline \multirow[t]{3}{*}{$17 / 07 / 2008$} & $12: 14$ & 263 & 5 & 230 & 493 & 53.35 & 2.44 & 20.48 \\
\hline & $14: 30$ & 262 & 1 & 231 & 493 & 53.14 & 1.9 & 22.38 \\
\hline & $15: 00$ & 257 & 5 & 236 & 493 & 52.13 & 0.3 & 22.68 \\
\hline \multirow[t]{4}{*}{$18 / 07 / 2008$} & $11: 00$ & 245 & 3 & 248 & 493 & 49.70 & 2 & 24.68 \\
\hline & $12: 15$ & 243 & 2 & 250 & 493 & 49.29 & 15 & 26.18 \\
\hline & $12: 35$ & 242 & 1 & 251 & 493 & 49.09 & 0.3 & 26.48 \\
\hline & $15: 10$ & 240 & 2 & 253 & 493 & 48.68 & 2.35 & 28.83 \\
\hline & idice de & urez & & Calibre & $e=118$ & & & \\
\hline
\end{tabular}

A partir de los resultados de los cuadros de secado bajo diferentes condiciones se construyó el Cuadro 7

Cuadro $\mathrm{N}^{0} 7$ Resultados del Proceso de Secado de aceituna

\begin{tabular}{|c|c|c|}
\hline $\begin{array}{l}\text { Temperatura } \\
\text { del aire } \\
\left({ }^{\circ} \mathrm{C}\right) \\
\end{array}$ & $\begin{array}{l}\text { Velocidad } \\
\text { del aire } \\
(\mathrm{m} / \mathrm{s}) \\
\end{array}$ & $\begin{array}{l}\text { Velocidad } \\
\text { de secado } \\
\text { (gr/gr. ms. h) }\end{array}$ \\
\hline 70 & 0.5 & 0.04516 \\
\hline 60 & 1.5 & 0.02886 \\
\hline 65 & 1 & 0.04421 \\
\hline 60 & 0.5 & 0.02459 \\
\hline 70 & 1.5 & 0.04578 \\
\hline
\end{tabular}

PROCESO DE OPTIMIZACIÓN DEL PROCESO DE SECADO DE ACEITUNA NEGRA

Utilizando los parámetros presentados en el Cuadro 7 se ha realizado el análisis y su proceso de optimización del proceso de secado utilizando el Software Statgraphics 5.1

En la Figura 5 se observa que la temperatura del aire de secado tiene más impacto en el secado de la aceituna, con una menor significancia como se observa en la misma figura, la velocidad del aire

\section{Gráfico de Pareto estandarizado para vel secado}

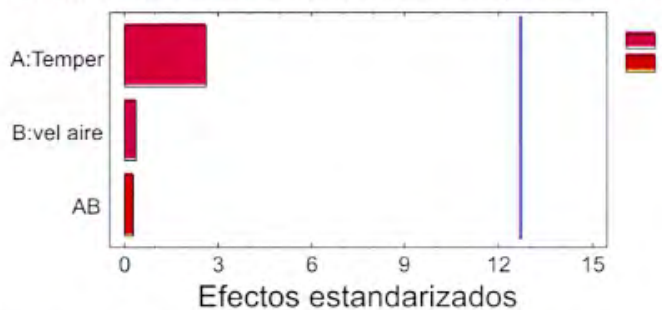

Figura 5 Grafico de Pareto estandarizado para velocidad de secado

La interacción entre ambos parámetros no tiene significancia o relevancia.

El software nos indica en cuanto al la maximización velocidad de secado lo siguiente

Valor Optimo $=0.0474055$

\begin{tabular}{lccc} 
Factor & Inferior & Mayor & Óptimo \\
\hline Temperatura & 60,0 & 70,0 & 70,0 \\
Velocidad aire & 0,5 & 1,5 & 1,5
\end{tabular}

Lo que indica óptimo inicialmente lo considera como 70 grados centígrados y una velocidad de $1.5 \mathrm{~m} / \mathrm{s}$

Para un mejor interpretación de resultados se realizó un análisis de varianza como se muestra a continuación en el Anexo 1.

Analizando el Cuadro 8, podemos indicar que existen diferencias entre las temperaturas utilizadas.

De Figura 6 nos muestra al impacto de cada variable de entrada al proceso de secado, por lo que podemos indicar que la temperatura tiene un mayor impacto en la velocidad de secado y la velocidad del aire un menor efecto en la velocidad de secado

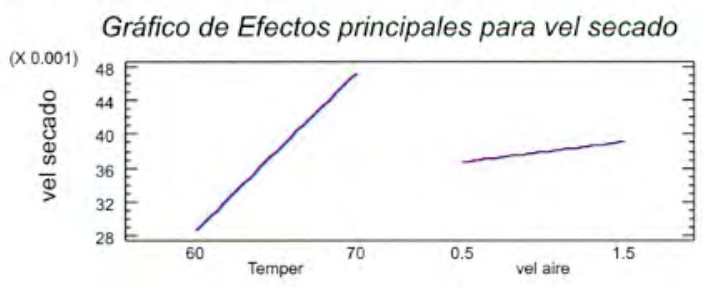

Figura 6 Efectos Principales para la velocidad secado. 
El proceso de optimización de las variables que se utilizaron para desarrollar este proceso de secado se muestran en la Figura 7

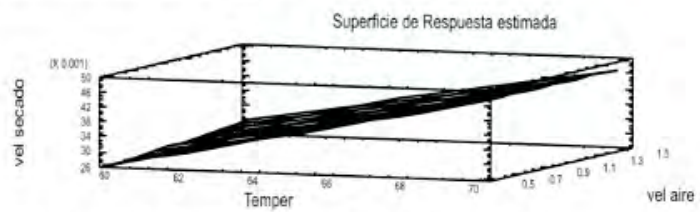

Gráfico / Superficie de respuesta estimada

Donde se visualiza lo anteriormente expresado en cuanto a la importancia de la temperatura del aire

Para obtener una mejor visión de la optimización se preparó el contorno de la superficie de repuesta, el mismo que se muestra en la figura 8 , donde se puede delimitar el área donde se presentan los mejores resultados esa corresponde entre 69-69.5 grados centígrados y una velocidad del aire entre $0.5-1.5 \mathrm{~m} / \mathrm{s}$, lo que indica poca variabilidad con respecto al este parámetro.

En la Figura 9 se observa el resultado del proceso de escalamiento optimización del presente software, donde ese puede observar que el óptimo se encuentra en el área de 69 grados centígrados y luego se mantiene constante y respecto al la velocidad del aire se muestra estable y sin mucha variación.

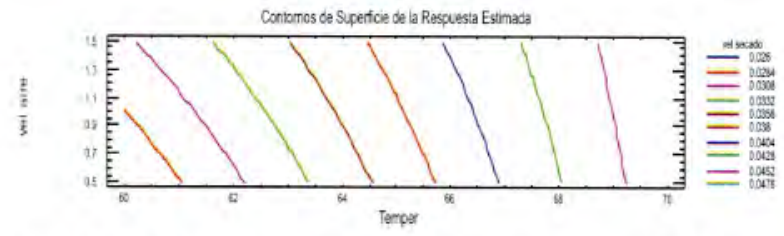

Figura 8 Contorno de superficie de la repuesta estimada.

Superficie de Respuesta estimada

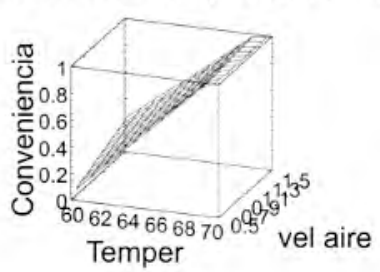

Figura9 Superficiederespuesta estimada

\section{CONCLUSIONES}

1. La temperatura del aire de secado resultó el parámetro mas importante en el proceso de deshidratación de la aceituna, y se consideró el valor óptimo de 68 grados centígrados, para los valores de indice de madurez entre 4,52-5, con un calibre entre 94-152

2. La velocidad del aire tuvo un valor poco importante y se consideró como la óptima $0,5 \mathrm{~m} / \mathrm{s}$.

3. La velocidad de secado óptima fue $0,476 \mathrm{~kg}$ agua $/ \mathrm{kg}$ de materia seca.h
4. La interacción de estos parámetros velocidad del aire y temperatura no fue de importancia relevante.

5. La apariencia de las aceitunas secas fue de características muy similares .

\section{RECOMENDACIONES}

1. Realizar ensayos a distintos niveles de madurez.

2. Determinar los parámetros óptimos para el secado utilizando sal en la primera parte del secado.

3. Determinar el coeficiente de difusibilidad del agua durante el secado de la aceituna. deshidratación

\section{REFERENCIAS BIBLIOGRAFICAS}

ANPEAP (1988). "Estructura competitiva en aceituna", obtenido de www. mincetur gob.pe.el 04 de marzo de 2009.

Boskou, D (1998).Quimica y Tecnología de aceite de oliva. Editorial Mundi.

Brennam; etal. (1980) Las operaciones en la ingeniería de los alimentos. Editorial Acribia, Zaragoza España.

Casilla, Barman (2006). "Situación actual y perspectivas de mercado de aceitunas y aceite de oliva". Presentación PowerPoint.

CODEX ALIMENTARIUS ( 1981). "Norma CODEX para aceituna de mesa, bajado de www.codexalimentarius.net

Cabieses, Fernanado: 100 años de pan.

Charm S. Food Enginiering. AVI. Wesport.

Díaz, A. (1988). Diagnóstico de la producción de aceitunas de mesa en el Perú.

Earle R, L. (1968). Ingeniería de los alimentos. Editorial Acribia, Zaragoza, España.

Gambilla, et al. (2000). Effect of different on drying of green tables olives (Ascolana tenera var). Revista Grasas y Aaceite Vol. 51, Fac. 3173.

Infante, Isabel (2008). IPAFA. Diseño y desarrollo de producto comercial en base a aceitunas prietas", bajado de www.juntadeandalucia .es

Ministerio de Agricultura (20089. Bajado de www.portalagrario.com.pe

"Olivar super intensivo". (2009), obtenido en www.olivar superintensivo.com, el dia 26 de marzo del 2009.

Savarredi Eugenio(1988). Manual de preparación de aceitunas. Fondo de Cooperación Técnica PeruanoArgentina.

Single,R; Heldman,D(1993). Introducción a la ingeniería de alimentos. .Editorial Acribia, Zaragoza,. España. 